\title{
ON SIMPLE QUADRATURES
}

\author{
PHILIP DAVIS
}

The trapezoidal rule and the parabolic rule so frequently employed in computational work hold without error for the classes of linear and quadratic functions respectively. More generally, if $n+1$ distinct points $\lambda_{0}, \lambda_{1}, \cdots, \lambda_{n}$ are given, then it is possible to find an integration formula

$$
\int_{0}^{1} f(x) d x=\sum_{i=0}^{n} a_{i} f\left(\lambda_{i}\right)
$$

which will be exact for the class $P_{n}$ of polynomials of degree at most $n$, and where, furthermore, the weights $a_{i}$ depend merely on the abscissas $\lambda_{i}$ and not on the particular function of the class $P_{n}$ which has been selected. To find such a formula, we need only to construct the Lagrange interpolation polynomial for the abscissas $\lambda_{i}$, and then integrate. More precisely, we have

$$
f(x)=\sum_{i=0}^{n} f\left(\lambda_{i}\right) \frac{A(x)}{\left(x-\lambda_{i}\right) A^{\prime}\left(\lambda_{i}\right)} ; \quad A(x)=\prod_{i=0}^{n}\left(x-\lambda_{i}\right),
$$

$$
f(x) \in P_{n},
$$

and therefore

$$
\begin{array}{r}
\int_{0}^{1} f(x) d x=\sum_{i=0}^{n} a_{i} f\left(\lambda_{i}\right) ; \quad a_{i}=\int_{0}^{1} A(x) d x /\left(x-\lambda_{i}\right) A^{\prime}\left(\lambda_{i}\right), \\
f(x) \in P_{n} .
\end{array}
$$

It is clear that the coefficients $a_{i}$, generally known as the Cotes numbers, depend merely on the $\lambda_{i}$.

The existence of exact quadrature formulas of the type (3) suggests the following problem. Suppose that there is given a more or less extensive class $S$ of functions which are Riemann integrable over $[0,1]$. Does there exist a quadrature of the form

$$
\int_{0}^{1} f(x) d x=\sum_{i=0}^{\infty} a_{i} f\left(\lambda_{i}\right)
$$

which is valid for all functions of the class $S$ ? The abscissas $\lambda_{i}$ are assumed to be distinct and fixed, while the weights $a_{i}$ are to be independent of the particular $f \in S$ chosen. If a formula (4) exists, valid

Received by the editors April 24, 1952. 
for all $f \in S$, then we shall say that the class $S$ possesses a simple quadrature. This terminology will serve to distinguish the scheme (4) from the more general and frequently investigated ${ }^{1}$ quadrature scheme

$$
\int_{0}^{1} f(x) d x=\lim _{n \rightarrow \infty} \sum_{k=0}^{n} a_{n k} f\left(\lambda_{n k}\right) .
$$

If, in addition, there is a quadrature (4) for which

$$
\sum_{i=0}^{\infty}\left|a_{i}\right|<\infty \text {, }
$$

then we shall say that the class $S$ possesses an absolutely convergent simple quadrature.

In the present note, we investigate this possibility for a number of familiar classes of functions and under a number of assumptions as to the distribution of the abscissas. We shall find that the following general situation prevails: a simple quadrature exists if the class $S$ contains "relatively few" functions, while there can be no simple quadrature if $S$ contains "too many" functions. Thus, for example, a quadrature exists for each of the classes $P_{n}$ and, as we shall see, for the class $P$ of all polynomials, but not for the class of Riemann integrable functions or for the class of functions merely continuous in $[0,1]$.

In dealing with the classes of Riemann integrable or of continuous functions on $[0,1]$, if we are to have any success in finding a simple quadrature, then the abscissas must be chosen so as to be everywhere dense in $[0,1]$. For if this is not the case, then starting with a function $f$ for which (4) holds, we may alter its values interior to an interval which is free of $\lambda$ 's thereby altering the value of the integral without altering the value of the right-hand sum. But even with this precaution, these classes are too large to admit a simple quadrature. In dealing with classes of polynomials or of analytic functions we are free to relax this condition on the abscissas.

THEOREM 1. The class of functions which are Riemann integrable over $[0,1]$ does not possess a simple quadrature.

Proof. Suppose the contrary. Not all the weights $a_{i}$ can equal zero as can be seen by choosing $f(x)=1$. Suppose, then, that $a_{k} \neq 0$ for some $k$. Define a function $f(x)$ as follows:

$$
f(x)=0, \quad x \neq \lambda_{k},
$$$$
f(x)=1,
$$$$
x=\lambda_{k} .
$$

\footnotetext{
${ }^{1}$ Cf., e.g., P6lya [4], Szegö [6], Feldheim [2].
} 
It is clear that $f(x)$ is integrable to the value 0 , whereas the righthand side of (4) reduces to $a_{k} \neq 0$. Thus we arrive at a contradiction.

The same conclusion holds for the more restricted class of continuous functions. The proof is based upon a refinement of the simple argument given above. The construction used in Theorem 3 is a modification of one due to Lebesgue [3]. (Cf. also Pólya [4] and Feldheim [2].) We shall find it convenient to establish a preliminary theorem.

TheOREM 2. The class of functions continuous on $[0,1]$ does not possess an absolutely convergent simple quadrature.

Proof. Assume the contrary. As above, let $a_{k} \neq 0$ for some $k$. We may suppose, in addition, that $0<\lambda_{k}<1$. Designate by $I_{\epsilon}$ the interval $\lambda_{k}-\epsilon \leqq x \leqq \lambda_{k}+\epsilon$. For $\epsilon$ sufficiently small, the abscissas $\lambda_{i}$ lying in $I_{\epsilon}$ will form a subset of the set of all abscissas, and of the former, $\lambda_{k}$ will possess the minimum subscript. In the order of increasing subscripts we shall have, say, $\lambda_{k}=\lambda_{n_{1}(\epsilon)}, \lambda_{n_{2}(\epsilon)}, \cdots$, where, moreover, $\lim _{\epsilon \rightarrow 0} n_{2}(\epsilon)=\infty$. For each $\epsilon$ construct the following continuous triangular shaped function

$$
\begin{array}{rlrl}
f_{\epsilon}(x) & =0, & 0 \leqq x \leqq \lambda_{k}-\epsilon, \\
f_{\epsilon}(x) & =\text { linear, } & \lambda_{k}-\epsilon \leqq x \leqq \lambda_{k}, \\
f_{\epsilon}\left(\lambda_{k}\right) & =1, & & \\
f_{\epsilon}(x) & =\text { linear, } & \lambda_{k} \leqq x \leqq \lambda_{k}+\epsilon, \\
f_{\epsilon}(x) & =0, & \lambda_{k}+\epsilon \leqq x \leqq 1 .
\end{array}
$$

In particular, we have $\int_{0}^{1} f_{\epsilon}(x) d x=\epsilon$ and $\left|f_{\epsilon}(x)\right| \leqq 1$. Thus by (4) and (8), we have

$$
\epsilon=\sum_{j=0}^{\infty} a_{j} f_{\epsilon}\left(\lambda_{j}\right)=a_{k} f_{e}\left(\lambda_{k}\right)+\sum_{j=2}^{\infty} a_{n_{j}(\epsilon)} f_{\epsilon}\left(\lambda_{n_{j}(\epsilon)}\right) .
$$

Whence,

$$
\left|\epsilon-a_{k}\right| \leqq \sum_{j=2}^{\infty}\left|a_{n_{j}(\epsilon)}\right|
$$

Now as $\epsilon \rightarrow 0$, the left side of (9) approaches $\left|a_{k}\right| \neq 0$, whereas the right side approaches zero. Thus we have a contradiction.

ThEOREM 3. The class of all functions continuous on $[0,1]$ does not possess a simple quadrature.

Proof. Assume the contrary. Let $A_{n}=\sum_{j=0}^{n}\left|a_{j}\right|$. If $\lim _{n \rightarrow \infty} A_{n}<\infty$, 
then the class of functions continuous on $[0,1]$ would possess an absolutely convergent simple quadrature, and this is not possible by the previous theorem. We may therefore assume that $\lim _{n \rightarrow \infty} A_{n}=\infty$. We may further suppose that no $a_{j}$ vanishes, for if it does, we need merely suppress that $\lambda_{j}$.

For each integer $k(k=0,1,2, \cdots)$, define a continuous function $f_{k}(x)$ by the following requirements

$$
f_{k}\left(\lambda_{j}\right)=a_{j} /\left|a_{j}\right|=\operatorname{sgn} a_{j} \quad(j=0,1,2, \cdots, k),
$$

(10) $f_{k}(x)$ is to vary linearly in the intervals between the abscissas $\lambda_{0}, \lambda_{1}, \cdots, \lambda_{k}$ and to be constant over the end intervals.

It is clear that for each $k$ we have

$$
\begin{array}{lr}
\left|f_{k}(x)\right| \leqq 1, & 0 \leqq x \leqq 1, \\
\sum_{j=0}^{k} a_{j} f_{k}\left(\lambda_{j}\right)=A_{k} & (k=0,1,2, \cdots) .
\end{array}
$$

We next define a sequence of integers $k_{j}(j=1,2, \cdots)$ by an inductive process. Let $k_{1}=1$. Having defined $k_{1}, k_{2}, \cdots, k_{n-1}$, we define $k_{n}$ as follows. We consider the function

$$
h_{n-1}(x)=f_{k_{1}}(x) / 3+f_{k_{2}}(x) / 3^{2}+\cdots+f_{k_{n-1}}(x) / 3^{n-1} .
$$

The function $h_{n-1}(x)$ is continuous on $[0,1]$ and therefore, by our quadrature hypothesis, $\sum_{j=0}^{\infty} a_{j} h_{n-1}\left(\lambda_{j}\right)$ converges, and its partial sums are therefore bounded:

$$
\left|\sum_{j=0}^{p} a_{j} h_{n-1}\left(\lambda_{j}\right)\right| \leqq M_{n-1} \quad(p=0,1,2, \cdots) .
$$

Now select $k_{n}$ so large that

$$
\begin{aligned}
k_{n} & >k_{n-1}, \\
A_{k_{n}} & >2 \cdot 3^{n}\left(M_{n-1}+n\right) .
\end{aligned}
$$

Condition (14b) can be satisfied inasmuch as $\lim _{n \rightarrow \infty} A_{n}=\infty$. Having thus defined the sequence of integers $k_{n}$, we consider the function

$$
h(x)=\lim _{n \rightarrow \infty} h_{n}(x)=\sum_{n=1}^{\infty} f_{k_{n}}(x) / 3^{n} .
$$

Because of (11a), this series converges uniformly and absolutely in $[0,1]$, and hence $h(x)$ is continuous over that interval. Now, 


$$
\begin{aligned}
\sum_{j=0}^{k_{n}} a_{j} h\left(\lambda_{j}\right)=\sum_{j=0}^{k_{n}} a_{j} h_{n-1}\left(\lambda_{j}\right) & +\frac{1}{3^{n}} \sum_{j=0}^{k_{n}} a_{j} f_{k_{n}}\left(\lambda_{j}\right) \\
& +\sum_{j=0}^{k_{n}} a_{j}\left(\frac{f_{k_{n+1}}\left(\lambda_{j}\right)}{3^{n+1}}+\frac{f_{k_{n+2}}\left(\lambda_{j}\right)}{3^{n+2}}+\cdots\right)
\end{aligned}
$$

so that by (13), (11b), and (11a),

$$
\begin{aligned}
\left|\sum_{j=0}^{k_{n}} a_{j} h\left(\lambda_{j}\right)\right| & \geqq-M_{n-1}+\frac{A_{k_{n}}}{3^{n}}-A_{k_{n}}\left(\frac{1}{3^{n+1}}+\frac{1}{3^{n+2}}+\cdots\right) \\
& =\frac{A_{k_{n}}}{2 \cdot 3^{n}}-M_{n-1}>n,
\end{aligned}
$$

the last inequality following from (14b). If we now let $n \rightarrow \infty$, we see that the quadrature scheme does not even converge when applied to the continuous function $h(x)$. This contradicts our assumption.

From the fairly wide class of continuous functions we turn now to the fairly restricted class $P$ of all polynomials. This class cannot be treated by the device (2), yet it possesses an absolutely convergent simple quadrature. We shall prove this by using a theorem on infinite systems of linear equations due to Pólya [5], which we now state for convenience of reference.

Theorem (Pólya). Let there be given an infinite system of linear equations in infinitely many unknowns $x_{i}$ :

$$
\begin{aligned}
& a_{00} x_{0}+a_{01} x_{1}+a_{02} x_{2}+\cdots=b_{0}, \\
& a_{10} x_{0}+a_{11} x_{1}+a_{12} x_{2}+\cdots=b_{1},
\end{aligned}
$$

Let the coefficients $a_{j k}$ be subjected to the following conditions

$$
\lim _{k \rightarrow \infty} a_{j-1, k} / a_{j, k}=0 \quad(j=1,2, \cdots)
$$

and suppose that

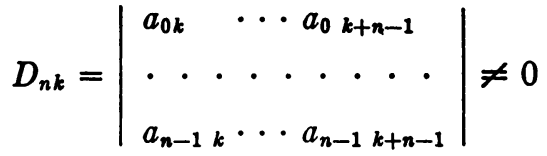

$$
\begin{aligned}
& (n=1,2, \cdots ; k=0,1,2, \cdots) \text {. }
\end{aligned}
$$

The constants $b_{k}$ may be completely arbitrary. Then there exists a solution $x_{i}$ of the system (16) which is such that all the series in (16) converge absolutely. 
Conditions (17) and (18) may be relaxed. In particular, (17) may be replaced by

$$
\begin{array}{r}
\liminf _{k \rightarrow \infty}\left[\left|a_{0 k}\right|+\left|a_{1 k}\right|+\cdots+\left|a_{j-1 k}\right|\right] /\left|a_{j k}\right|=0 \\
(j=1,2, \cdots) .
\end{array}
$$

The solution to (16) is not unique under these conditions, and infinitely many such solutions may be found by a step by step process. Let us now apply this theorem to the problem at hand.

Theorem 4. The class $P$ of all polynomials possesses absolutely convergent simple quadratures.

Proof. Select $\lambda_{0}<\lambda_{1}<\lambda_{2}<\cdots$ and $\lim _{n \rightarrow \infty} \lambda_{n}=\infty$. It is clearly sufficient to show that the functions $x^{n}(n=0,1, \cdots)$ can be integrated by a quadrature (4) with these abscissas. We must therefore have

$$
1 /(n+1)=\int_{0}^{1} x^{n} d x=\sum_{j=0}^{\infty} a_{j} \lambda_{j}^{n} \quad(n=0,1,2, \cdots) .
$$

Regarding (20) as a system of linear equations in the unknowns $a_{j}$, and comparing (20) with (16), we have $a_{j k}=\lambda_{k}^{j}$. Therefore, $\lim _{k \rightarrow \infty} a_{j-1} / a_{j k}=\lim _{k \rightarrow \infty} 1 / \lambda_{k}=0$. Furthermore,

$$
\begin{array}{r}
D_{k n}=\left|\begin{array}{cccc}
1 & 1 & \cdots & 1 \\
\lambda_{k} & \lambda_{k+1} & \cdots & \lambda_{k+n-1} \\
\cdot \cdot & \cdot & \cdot & \cdot \\
\lambda_{k}^{n-1} & \lambda_{k+1}^{n-1} & \cdots & \lambda_{k+n-1}^{n-1}
\end{array}\right|=\prod_{i>j}\left(\lambda_{k+i}-\lambda_{k+j}\right) \neq 0 \\
(i, j=0,1, \cdots, n-1) .
\end{array}
$$

The conditions of the previous theorem are therefore satisfied, so that there is a solution $a_{i}$ of (20) for which each series converges absolutely.

TheOREM 5. The class $P$ of all polynomials possesses an absolutely convergent simple quadrature with abscissas $\lambda_{k}$ if and only if

$$
\limsup _{k \rightarrow \infty}\left|\lambda_{k}\right|=\infty \text {. }
$$

Proof. Suppose first that (21) holds. Then for some subsequence $\lambda_{k_{n}}$ we have $\lim _{n \rightarrow \infty}\left|\lambda_{k_{n}}\right|=\infty$. Referring now to (20) and to the weaker condition (19), let us consider

$$
R_{k j}=\left\{1+\left|\lambda_{k}\right|+\left|\lambda_{k}\right|^{2}+\cdots+\left|\lambda_{k}\right|^{j-1}\right\} /\left|\lambda_{k}\right|^{j} .
$$


For $n$ sufficiently large, we have $\left|\lambda_{k_{n}}^{j-1}\right|>\left|\lambda_{k_{n}}\right|^{p}(p=0,1, \cdots, j-2)$ so that $R_{k_{n} j} \leqq(j)\left|\lambda_{k}^{j-1}\right| /\left|\lambda_{k}^{j}\right|=j /\left|\lambda_{k_{n}}\right|$. Thus, for $j=1,2, \cdots$, we have $\lim _{n \rightarrow \infty} R_{k_{n} j}=0$, and from this it follows that $\lim \inf _{k \rightarrow \infty} R_{k j}=0$ $(j=1,2, \cdots)$. There therefore exist absolutely convergent solutions of (20), and hence the class $P$ possesses an absolutely convergent simple quadrature with the abscissas $\lambda_{k}$.

Conversely, we shall show that the existence of an absolutely convergent quadrature for the set $P$ with abscissas $\lambda_{k}$ for which $\lim \sup _{k \rightarrow \infty}\left|\lambda_{k}\right|<\infty$ leads to a contradiction. Under this hypothesis, let $\sigma_{1}=$ g.l.b. $\lambda_{k}, \sigma_{2}=$ l.u.b. $\lambda_{k}$. Then $-\infty<\sigma_{1}<\sigma_{2}<\infty$. We now distinguish two cases depending upon the relative position of the two intervals $I:[0,1]$ and $S:\left[\sigma_{1}, \sigma_{2}\right]$.

We consider first the case where the interval $I$ is not contained in $S$. We shall show that this leads us to a simple quadrature for the set of functions continuous over $S$. This will contradict Theorem 3 inasmuch as this theorem can be stated for an arbitrary interval. Since $I$ is not contained in $S$, the set $I-S$ is not empty and consists of one or two intervals. Denote by $I^{\prime}$ the interval $\min \left(0, \sigma_{1}\right) \leqq x \leqq \max \left(1, \sigma_{2}\right)$. Let $f(x)$ be continuous on $S$. Then we may surely find a function $f^{*}(x)$ which is continuous on $I^{\prime}$ and has the following properties

$$
\begin{array}{cc}
f^{*}(x)=f(x), & x \in S, \\
\int_{0}^{1} f^{*}(x) d x=\int_{\sigma_{1}}^{\sigma_{2}} f^{*}(x) d x=\int_{\sigma_{1}}^{\sigma_{2}} f(x) d x . &
\end{array}
$$

Let now $\epsilon>0$ be given. Then by Weierstrass' theorem, we may find a polynomial $P(x)$ such that $\left|f^{*}(x)-P(x)\right|<\epsilon, x \in I^{\prime}$. Consider now

$$
\begin{aligned}
\int_{0_{1}}^{\sigma_{2}} f(x) d x-\sum_{n=0}^{\infty} a_{n} f\left(\lambda_{n}\right)= & \int_{0}^{1} f^{*}(x) d x-\int_{0}^{1} P(x) d x \\
& +\sum_{n=0}^{\infty} a_{n} P\left(\lambda_{n}\right)-\sum_{n=0}^{\infty} a_{n} f\left(\lambda_{n}\right) .
\end{aligned}
$$

Thus,

$$
\begin{aligned}
\left|\int_{\sigma_{1}}^{\sigma_{2}} f(x) d x-\sum_{n=0}^{\infty} a_{n} f\left(\lambda_{n}\right)\right| \leqq & \int_{0}^{1}\left|f^{*}(x)-P(x)\right| d x \\
& +\sum_{n=0}^{\infty}\left|a_{n}\right|\left|P\left(\lambda_{n}\right)-f\left(\lambda_{n}\right)\right| \\
\leqq & \epsilon\left(1+\sum_{n=0}^{\infty}\left|a_{n}\right|\right) .
\end{aligned}
$$


By letting $\leftrightarrow \rightarrow 0$ we have $\int_{\sigma_{1}}^{\sigma_{2}} f(x) d x=\sum_{n=0}^{\infty} a_{n} f\left(\lambda_{n}\right)$ holding for all $f$ continuous on $S$.

We next consider the case where the interval $I$ is contained in $S$. In this case we can show that there is a simple quadrature for the class of functions continuous on $[0,1]$. This will again be a contradiction. More precisely, let $N_{1}$ designate the set of subscripts $\boldsymbol{n}$ for which $\lambda_{n} \in I$. We shall show that $\int_{0}^{1} f(x) d x=\sum_{i \in N_{1}} a_{i} f\left(\lambda_{i}\right)$ for all $f$ continuous on $[0,1]$. Inasmuch as it has been assumed that $\sum_{i=0}^{\infty}\left|a_{i}\right|<\infty$, the convergence of the former series is absolute, and the order of the terms is immaterial. Let $f(x)$ be continuous on $I$. For each sufficiently small $\epsilon>0$, define a continuous function $f_{\mathrm{e}}(x)$ as follows:

$$
\begin{array}{lr}
f_{\epsilon}(x)=f(x), & x \in I, \\
f_{\epsilon}(x)=0 & \text { for } \sigma_{1} \leqq x \leqq-\epsilon \text { and for } 1+\epsilon \leqq x \leqq \sigma_{2}, \\
f_{\epsilon}(x) \text { linear } & \text { in }-\epsilon \leqq x \leqq 0 \text { and in } 1 \leqq x \leqq 1+\epsilon .
\end{array}
$$

Designate by $N_{2}$ the set of subscripts $n$ for which $\lambda_{n}$ lies in $\sigma_{1} \leqq x \leqq-\epsilon$ or in $1+\epsilon<x \leqq \sigma_{2}$, and by $N_{2}$ the set for which $\lambda_{n}$ lies in $-\epsilon \leqq x<0$ or in $1<x \leqq 1+\epsilon$. Note that if $n_{\mathrm{e}}=\min _{n \in N_{8}} n$, then $\lim _{\mathrm{c} \rightarrow 0} n_{\mathrm{e}}=\infty$. Now let

$$
P(x)=P(x, \epsilon)
$$

be a polynomial which approximates $f_{e}(x)$ uniformly on $S$ to within $\epsilon$ :

$$
\left|f_{c}(x)-P(x)\right|<\epsilon, \quad x \in S .
$$

We have

$$
\int_{0}^{1} P(x) d x=\sum_{n=0}^{\infty} a_{n} P\left(\lambda_{n}\right)=\sum_{n \in N_{1}}+\sum_{n \in N_{2}}+\sum_{n \in N_{2}} a_{n} P\left(\lambda_{n}\right)
$$

inasmuch as we have assumed the existence of an absolutely convergent quadrature for the class $P$. But

$$
\begin{aligned}
\int_{0}^{1} f(x) d x-\sum_{n \in N_{1}} a_{n} f\left(\lambda_{n}\right)= & \int_{0}^{1} f(x) d x-\int_{0}^{1} f_{6}(x) d x+\int_{0}^{1} f_{c}(x) d x \\
& -\int_{0}^{1} P(x) d x+\sum_{n \in N_{1}} a_{n} P\left(\lambda_{n}\right) \\
& +\sum_{n \in N_{2}} a_{n} P\left(\lambda_{n}\right)+\sum_{n \in N} a_{n} P\left(\lambda_{n}\right) \\
& -\sum_{n \in N_{1}} a_{n} f\left(\lambda_{n}\right)
\end{aligned}
$$

and therefore 


$$
\begin{aligned}
&\left|\int_{0}^{1} f(x) d x-\sum_{n \in N_{1}} a_{n} f\left(\lambda_{n}\right)\right| \\
& \leqq \int_{0}^{1}\left|f_{c}(x)-P(x)\right| d x+\sum_{n \in N_{1}}\left|a_{n}\right|\left|P\left(\lambda_{n}\right)-f\left(\lambda_{n}\right)\right| \\
&+\sum_{n \in N_{2}}\left|a_{n}\right|\left|P\left(\lambda_{n}\right)\right|+\sum_{n \in N_{3}}\left|a_{n}\right|\left|P\left(\lambda_{n}\right)\right| .
\end{aligned}
$$

Now for $n \in N_{2},\left|P\left(\lambda_{n}\right)\right| \leqq \epsilon$, while for all $x \in S$ and for $\epsilon$ sufficiently small $|P(x)| \leqq M, M=1+\max _{x \in I}|f(x)|$, so that

$$
\left|\int_{0}^{1} f(x) d x-\sum_{n \in N_{1}} a_{n} f\left(\lambda_{n}\right)\right| \leqq \epsilon+A \epsilon+A \epsilon+M \sum_{n=n_{e}}^{\infty}\left|a_{n}\right| ;
$$

$$
A=\sum_{n=0}^{\infty}\left|a_{n}\right|
$$

By letting $\epsilon \rightarrow 0$, it follows that $\int_{0}^{1} f(x) d x=\sum_{n \in N_{1}} a_{n} f\left(\lambda_{n}\right)$ which is a contradiction.

With regard to classes of functions which lie between the class $P$ of all polynomials and the class of continuous functions, we can indicate only several partial results. On the one hand it is clear, from Pólya's theorem, that there are classes of functions very much wider than $\boldsymbol{P}$ which admit an absolutely convergent simple quadrature. For instance, let $\left\{\mu_{n}\right\}$ be a sequence of exponents for which $\mu_{0}<\mu_{1}$ $<\cdots$ and $\lim _{n \rightarrow \infty} \mu_{n}=\infty$. Then $a_{j-1, k} / a_{j, k}=\left(\lambda_{k}\right)^{\mu_{j-1}-\mu_{j}}$ so that if $\lambda_{k} \rightarrow \infty$, condition (17) will be fulfilled, and the class of all finite combinations of powers $x^{\mu_{n}}$ will possess an absolutely convergent simple quadrature. On the other hand, it seems unlikely that a class as large as the class $E$ of all entire functions of exponential type can possess a simple quadrature. This is surely the case with absolutely convergent quadratures. For suppose that $E$ possessed an absolutely convergent quadrature, then we should have

$$
\frac{e^{i s}-1}{i s}=\int_{0}^{1} e^{i s x} d x=\sum_{n=0}^{\infty} a_{n} e^{i \lambda_{n \varepsilon}}, \quad s>0 .
$$

It follows ${ }^{2}$ from $\sum_{n=0}^{\infty}\left|a_{n}\right|<\infty$ that $\left(e^{i s}-1\right) /$ is is an almost periodic function. But this is clearly impossible inasmuch as $\lim _{e^{-\infty}}\left(e^{i \cdot}-1\right) /$ is $=0$.

A similar argument shows that the class $E_{\sigma}$ of entire functions of exponential type at most $\sigma, \sigma>0$, cannot possess a simple quadrature

2 Cf. Bohr [1, p. 52]. 
with abscissas $\lambda_{n}$ for which $0<\lambda_{0}<\lambda_{1}<\cdots, \lim _{n \rightarrow \infty} \lambda_{n}=\infty$. For suppose otherwise, the functions $e^{-s t} \in E_{\sigma}$ provided $|s| \leqq \sigma$. Therefore we should have

$$
\frac{1-e^{-s}}{s}=\sum_{n=0}^{\infty} a_{n} e^{-\lambda_{n} s}
$$

the Dirichlet series converging for $\operatorname{Re}(s)>-\sigma$. If we now set

$$
\alpha(t)=\sum_{\lambda_{k} \leqq t} a_{k}, \quad \alpha(t)=\frac{1}{2}\left\{\alpha\left(t^{+}\right)+\alpha\left(t^{-}\right)\right\}
$$

then we may write

$$
\frac{1-\sigma^{-s}}{s}=\int_{0}^{\infty} e^{-s t} d \alpha(t), \quad \operatorname{Re}(s)>-\sigma .
$$

But if a function $\beta(t)$ is defined by

$$
\begin{array}{rr}
\beta(t)=t & \text { for } 0 \leqq t \leqq 1 \\
\beta(t)=1 & \text { for } 1 \leqq t,
\end{array}
$$

then

$$
\frac{1-e^{-*}}{s}=\int_{0}^{\infty} e^{-x t} d \beta(t)
$$

so that by the uniqueness theorems for Laplace transforms $\alpha(t)=\beta(t)$ and hence $a_{k}$ must vanish for $k \geqq$ some $k_{0}$. This is clearly impossible.

\section{REFERENCES}

1. H. Bohr, Almost periodic functions, New York, 1947.

2. E. Feldheim, Theorie de la convergence des procedes d'interpolation et de quadrature mécanique, Mémorial des Sciences Mathématiques, no. 95, Paris, 1939.

3. H. Lebesgue, Sur les integrales singulières, Ann. Fac. Sci. Univ. Toulouse vol. 1 (1909) pp. 25-117.

4. G. P6lya, Über die Konvergenz von Quadraturverfahren, Math. Zeit. vol. 37 (1933) pp. 264-286.

5. - Eine einfache, mit funktiontheoretischen A ufgaben verknilbfte hinreichende Bedingung für die Auflösbarkeit eines Systems unendlich vieler linearer Gleichungen, Comment. Math. Helv. vol. 11 (1938-39) pp. 234-252.

6. G. Szegö, Orthogonal polynomials, Amer. Math. Soc. Colloquium Publications, vol. 23, New York, 1939.

MassachusetTs InSTITUTE of TeChNology 